Article

\title{
On the Computation of the Voltage Distribution along the Non-Linear Resistor of Gapless Metal Oxide Surge Arresters
}

\author{
Christos A. Christodoulou ${ }^{1}$, Vasiliki Vita ${ }^{1}$, Valeri Mladenov ${ }^{2}$ (D) and Lambros Ekonomou ${ }^{1,3, *}$ \\ 1 Department of Electrical and Electronic Engineering Educators ASPETE-School of Pedagogical and \\ Technological Education, N. Heraklion, 14121 Athens, Greece; christ_fth@yahoo.gr (C.A.C.); \\ vasvita@aspete.gr (V.V.) \\ 2 Department of Theoretical Electrical Engineering, Technical University of Sofia, "Kliment Ohridski" Blvd 8, \\ 1000 Sofia, Bulgaria; valerim@tu-sofia.bg \\ 3 Department of Electrical and Electronic Engineering, City, University of London, London EC1V 0HB, UK \\ * Correspondence: lambros.ekonomou.1@city.ac.uk; Tel.: +44-787-902-7320
}

Received: 24 September 2018; Accepted: 30 October 2018; Published: 6 November 2018

\begin{abstract}
The voltage distribution along the non-linear resistance of metal oxide surges is of great importance for their proper operation, since the non-uniform potential distribution results in higher thermal stresses of the varistor discs near the high voltage electrode, leading to a faster ageing of the discs at the top and, consequently, a downgrade in arrester effectiveness and reliability or even failures. The current work deals with the examination of the voltage distribution along the non-linear resistance of medium voltage metal oxide gapless surge arresters, using an appropriate computer tool, discussing configuration that improve the voltage distribution. Moreover, the impact of various factors on the voltage distribution is examined. The extracted results can contribute to the more efficient design of modern metal oxide gapless surge arresters, in an effort to ensure their reliable operation to protect the electrical equipment against lightning surges.
\end{abstract}

Keywords: surge arresters; electric field; voltage distribution; varistor

\section{Introduction}

Surge arresters are widely used in electrical installations, in order to improve their lightning performance, to protect the equipment of the system and reduce the annual failure overvoltage rate. Surge arresters are installed between phase and earth and behave as insulators for the normal operation of the system and as conductors for overvoltage conditions, due to their intense non-linear voltage-current characteristic. Gapless arresters have nowadays totally replaced the $\mathrm{SiC}$ gapped surge arresters, since they present more extreme nonlinearity of the V-I curve, rendering non necessary to disconnect the resistors form the line through serial spark gaps. Moreover, gapless arresters are innately faster-acting, considering that time delay due to series air gaps extinguishing the current is eliminated [1-3].

A critical issue for the appropriate performance of the gapless metal oxide surge arresters is the voltage distribution along their non-linear resistance (varistor). In an ideal situation, the varistor should be stressed uniformly, i.e., each resistor metal oxide block should be stressed by an equal part of the applied voltage. However, the voltage distribution along a metal oxide surge arrester is governed by the capacitances and the resistances of the non-linear resistors, the stray capacitances from the non-linear resistor column and metal flanges to earthed and live parts, and the boundary conditions (applied voltage, proximity and voltage applied to other objects in the vicinity). Stray capacitances result in uneven voltage distribution along the resistor column, with the maximum voltage stress 
typically appearing in the upper part of the arrester, closer to the high voltage electrode. The above phenomenon results in the ageing of these parts of the varistor, downgrading the appropriate function of the arresters, leading to possible failures and altering the voltage-current characteristic [3-6]. For these reasons, the uniform voltage distribution along the non-linear resistance is a critical issue for the designers and engineers, in order to achieve the most proper functioning of the arresters and limit the undesirable effects of the unequal stress of the varistor's parts.

Various works have been carried out for the voltage distribution along the nonlinear resistor of metal oxide arresters, in an effort to address to the above challenges. In [7] the finite element method is implemented, by using the ANSYS 7-1 computer tool, to examine the contribution of grading rings to the voltage distribution for $110 \mathrm{kV}$ gapless arresters. The extracted outcomes highlight the ageing and the downgrade of the varistor discs due to the non uniform voltage stress. In $[8,9]$ the impact of a surface pollution layer on the voltage distribution and the electric field of $15 \mathrm{kV}$ surge arresters are examined, using FEMLAB and PC Opera, respectively, since in [2] the voltage distribution for arresters with broken sheds is discussed. In [10], the electric field in conventional and novel built-in-bushing $\mathrm{ZnO}$ arresters is investigated by means of finite element modeling using COMSOL Multiphysics software, in an effort to mitigate the consequences of high potential gradients in the internal and the external of the arrester, in particular during transient conditions that may cause damages to the arrester's insulation, leading to a premature failure. In [11] the impact of the geometric characteristics and the placement position of the toroids on the distribution of the electric field around gapless arresters is examined by applying the SCSM (surface charge simulation method) method. The simulation outcomes indicate that the placement of the toroids at the $75-85 \%$ of the total height of the arresters result in the optimum voltage distribution along the non linear resistor. In [12] a semi-analytic finite element method is presented in an effort to handle the open boundary axisymmetric electrostatic field issues; the method is implemented for the estimation of the potential distribution of a high voltage gapless arrester with porcelain housing, showing a good agreement between the calculated and measured results. However, the methodology is complex and time-consuming and it is not supported by an already existing computer tool.

In this context, this paper proposes a procedure for the computation of the voltage distribution along medium voltage gapless surge arresters, by using an appropriate computer tool. The reliability of the simulation model is confirmed by comparing the estimated electric field around the arrester with experimental results. In addition, new design proposals for the improvement of the voltage distribution along the non linear resistance are discussed. Moreover, the impact of various factors (pollution on the surface of the external insulation, broken sheds) on the voltage distribution is examined. The extracted results can contribute to the more efficient design of modern metal oxide gapless surge arresters, in an effort to ensure their reliable operation to protect the electrical equipment against lightning surges.

\section{Voltage Distribution along the Non Linear Resistance of Metal Oxide Gapless Arresters}

The voltage distribution in a zinc oxide $(\mathrm{ZnO})$ surge arrester is regarded to be uneven, under normal operating conditions. The discs near the high voltage electrode are subjected to higher voltages and therefore thermal stresses, resulting in their fast thermal ageing. Hence, various attempts have been generally made to render the voltage distribution, which is determined by the geometry and the electrical properties of the materials used, as uniform as possible.

The voltage distribution can be evaluated by means of commonly available computer programs for calculation of electric fields and circuits. However, the extracted outcomes of such calculations are strongly influenced by the representations of the surge arrester and the prevailing boundary conditions [6].

Taking into account that the stray capacitances have a key role to the voltage distribution along the non-linear resistor column, the impact of various simplifications on the surge arrester model must be considered with respect to these capacitances. 
In details, the non-linear resistor column, including any metal spacers, should be represented by its actual dimensions and permittivity. An "equivalent" non-linear resistor column of larger diameter, and correspondingly decreased permittivity, results in a higher maximum voltage "equivalent" column without spacers, and with a correspondingly increased permittivity, also results in a higher maximum voltage stress. Moreover, the housing may be represented by a cylinder having an inner diameter equal to the inner diameter of the actual housing and radial thickness equal to the wall thickness of the actual housing. The permittivity should be that of the actual housing material, for example, porcelain or polymer. The sheds may be omitted since they have a negligible influence on the voltage distribution. As far as the material between the insulator and the non-linear resistor column is concerned, it should be modelled with its actual dimensions and permittivity. The metal flanges may be represented by cylinders having diameters equal to the maximum outer diameter of the actual flanges and heights equal to the heights of the actual flanges. The high-voltage lead should be represented by a vertical cylindrical conductor of a diameter not greater than the diameter of the actual line lead. Omitting the high-voltage lead results in a higher maximum voltage stress in the upper part of the arrester [6].

Figure 1 depicts a simplified multi-stage equivalent circuit of an arrester, which may be used with an electric circuit analysis program to determine the voltage distribution considering both capacitive and resistive effects. The arrester is modelled by the voltage-dependent resistances, the capacitances representing the non-linear resistor column and the stray capacitances to earth. Each stage of the equivalent circuit may represent one single metal oxide non-linear resistor, as the extreme case, or a section of the non-linear resistor column [6].

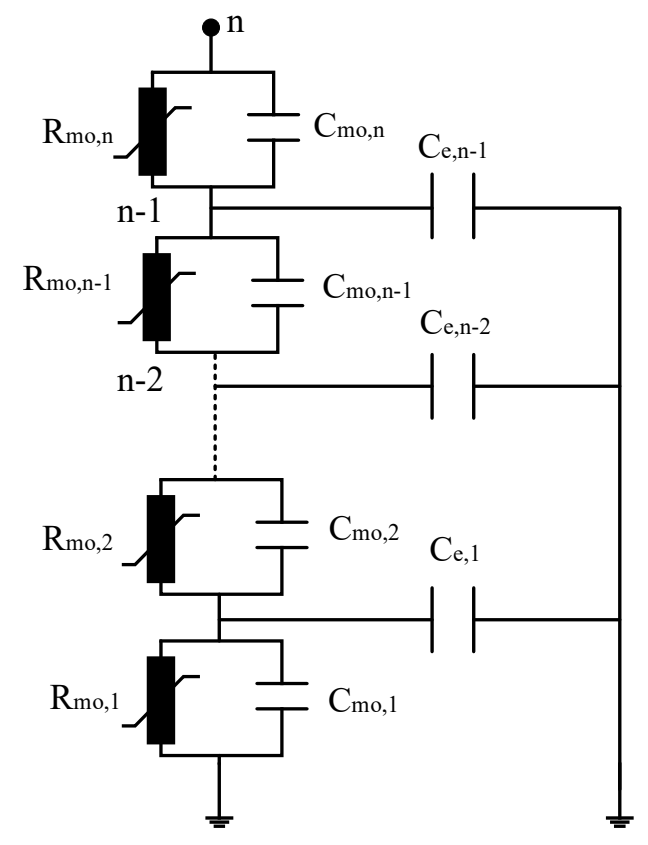

Figure 1. Simplified multi-stage equivalent circuit of an arrester $\left(R_{m o, x}\right.$ : voltage-dependent resistance of section $x, C_{m o, x}$ : capacitance of section $x, C_{e, x}$ : stray capacitance to earth at node $x, n$ : number of sections).

\section{Procedure for the Computation of the Voltage Distribution along the Non Linear Resistance of Metal Oxide Arresters}

The voltage distribution along the non-linear resistor of metal oxide surge arresters is difficult to measure, considering the structure of the arresters (the external insulation and the fiber glass enclose the varistor discs). Hence, the voltage distribution has to be estimated by simulation or theoretical procedures. An issue of priority that arises is how the accuracy of the implemented model can be ensured. In order to satisfy the above requirement, the appropriateness of the simulation model can be indirectly validated by comparing simulation and laboratory results of the electric field. Indeed, 
the electric field can be used as an index for the reliability of the simulation models. The proposed procedure is described as following:

Step 1: Design of the surge arrester model by using appropriate computer tool.

Step 2: Calculation of the electric field around the arrester

Step 3: Laboratory measurement of the electric filed around the arrester

Step 4: Comparison between simulation and experimental results

Step 5: In the case that the error is $<10 \%$, end; else, if the error is $>10 \%$ go to Step 1 and change the design parameters $(\varepsilon, \sigma$, geometry etc).

In previous works, the electric field around a metal oxide surge arrester was examined, by comparing experimental measurements with simulation results. In detail, the electric field around a typical medium voltage $\mathrm{ZnO}$ gapless polymeric housing surge arrester was measured $[3,13,14]$, using appropriate calibrated field meters $[15,16]$. The sensor was moved horizontally, at different distances along five axes, taking into consideration the geometry and the symmetry of the arrester, and at various heights $[3,13,14]$.

The electric field for given heights and distances is different for each axis, since it is influenced by the non-symmetrical geometry of the arrester. 2D simulation, ignoring these asymmetries, cannot represent these changes for each axis, so there is need for a 3D design of the sample. For this reason, the sample is designed and analyzed using 2D and 3D edition of the PC Opera, a Finite Element Analysis program [15,16], and the theoretical results are compared to the recorded measurements. Figure 2 depicts the 2D surge arrester model, designed in PC Opera, ignoring the non symmetrical parts of the sample and the test arrangement geometry. The dielectric permittivity is 100 for the varistor, 4.6 for the fiber glass, 3.9 for the external insulation and 1 for the air around the arrester. The conductivity is $10^{-14} \mathrm{~S} / \mathrm{m}$ for the fiber glass, $10^{-12} \mathrm{~S} / \mathrm{m}$ for the external insulation, 0 for the air and $10^{6} \mathrm{~S} / \mathrm{m}$ for the high voltage and earth electrodes. It is worth mentioning that since the varistor is a non linear element, its electrical conductivity is assigned according to the V-I characteristic during normal conditions. V-I curve is a valuable indicator that reveals the changes of resistance as a function of the voltage. Note that the varistors operate at most in the region where conduction is very small (near zero) [1]. In an effort to enhance the accuracy of the simulation outcomes, the density of the finite element mesh was increased in the critical regions inside and around the arrester, creating 60,212 elements and 120,925 nodes. In order to include the asymmetries of the high voltage and earth electrodes and of the total test arrangement configuration, the examined arrester was designed using the 3D edition of PC Opera (Figure 3). The electric characteristics of the materials are the same as in the 2D computations, since the mesh was, also, more dense in the critical regions inside and around the arrester, creating 1,323,551 elements and 226,167 nodes.

A good agreement has been achieved comparing the experimental with the simulation results. It is worth mentioning that the $3 \mathrm{D}$ model is more precise, due to the fact that takes into account the non symmetrical parts of the arrester and the experiment configuration. The $3 \mathrm{D}$ results, considering details of the designed parts of the arrester, are very close to the real recorded measurements.

The two designed models can be useful for the computation of the voltage distribution along the varistor, which cannot be measured, since it is impossible to reach the varistor inside the arrester. Via the used software the voltage distribution can be easily and precisely estimated, if the geometry of the sample is designed in an accurate way. The models can also be used to try changes of geometry or materials in order to achieve lower values of the electric field and more uniform voltage distribution of the arrester. 


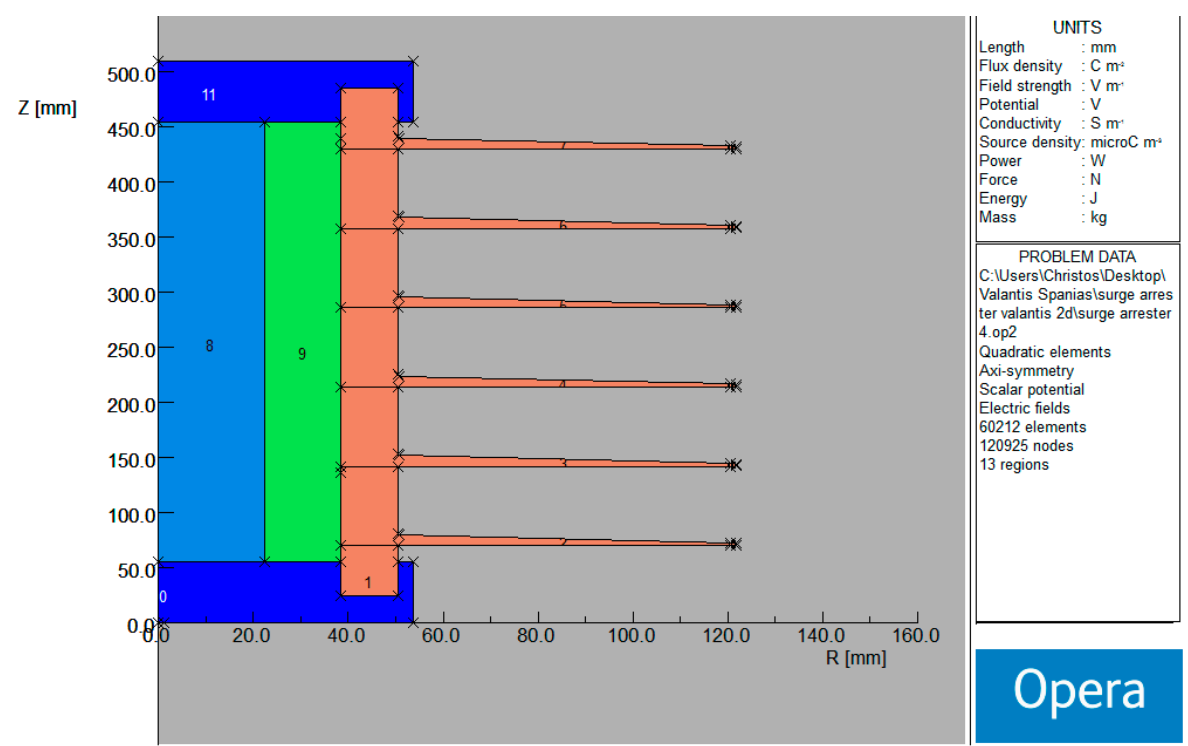

Figure 2. 2D surge arrester model.

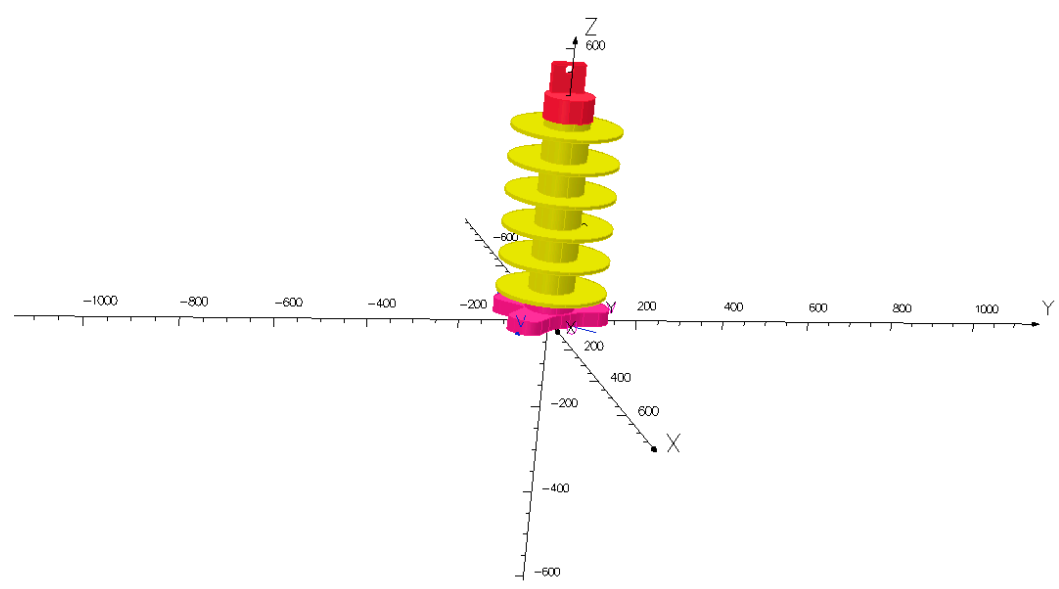

Figure 3. 3D surge arrester model.

From the above analysis there is a satisfactory convergence between experimental and theoretical results for the electric field around the surge arrester, which proves the appropriateness of the developed PC Opera models. By comparing the electric field simulation results to real recorded laboratory measurements it is concluded that the simulation procedure is reliable and can be leveraged for the evaluation of the voltage distribution along the varistor, which cannot be easily measured.

Figure 4 compares the voltage distribution along the varistor of the arrester, obtained from the 2D and 3D edition of PC Opera correspondingly, which is obviously not uniform, due to stray capacitances, with the maximum stress appearing in the upper part of the arrester. The obtained curves indicate that 2D and 3D models give similar results, so the 2D model, which is easier to be designed and handled, is adequate in an effort to examine the impact of various factors on the voltage distribution. 


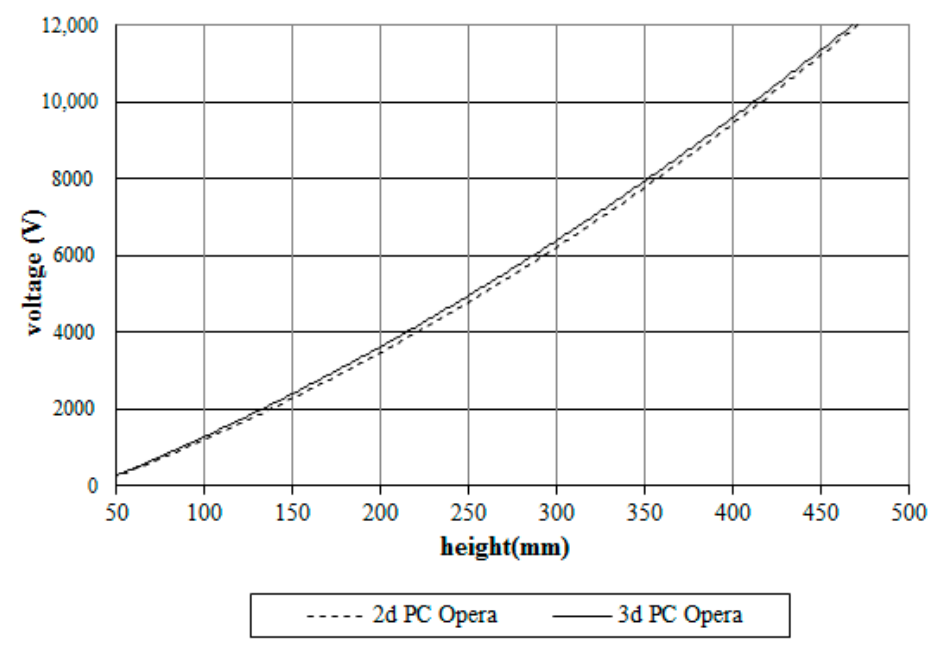

Figure 4. Comparison of the voltage distribution along varistor using 2D and 3D PC Opera.

\section{Design Proposals of the Varistor Geometry for the Improvement of Its Voltage Distribution}

The study of the voltage distribution along the non-linear resistance of metal oxide surge arresters on the steady state performance is of great importance (especially during the design procedure), since the non uniform potential distribution results to higher thermal stresses of the varistor discs near the high voltage electrode (due to the stray capacitances to ground), leading to a faster ageing of the discs at the top and, consequently, the downgrade of arresters' effectiveness and reliability or even failures. The improvement of the voltage distribution along the varistor is necessary, to accomplish the optimum utilization of the materials and to restrain the arresters' damages due to the excessive stresses. The voltage distribution in an arrester is strongly governed by the geometry of different components; in the next sections, various changes of the arresters geometry are presented for the enhancement of the potential distribution along the varistor.

\subsection{Uniform Increase of the Varistor'S Diameter}

Figure 5 presents the cutoff of the examined sample with a higher diameter of the non linear resistance $(4.8 \mathrm{~cm}$ instead of $4.3 \mathrm{~cm})$. Regions 1-7 correspond to the external insulation of the arrester, region 12 to the fiber glass, region 13 to the varistor, and regions 8 and 9 to the electrodes. The voltage distribution along the non linear resistance is depicted in Figure 8; the uniform increase of the varistor's diameter results in the improvement of the voltage distribution, becoming more uniform.

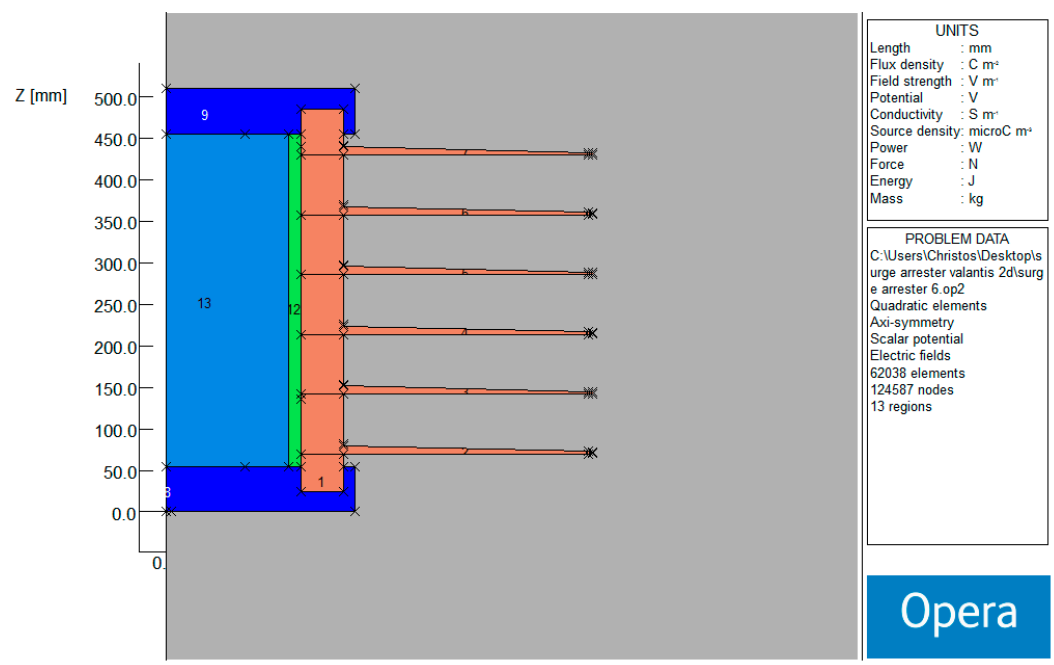

Figure 5. Arrester's model with increased varistor diameter. 


\subsection{Non Uniform Increase of the Varistor's Diameter}

The diameter of the non-linear resistance is higher near the grounding electrode $(4.8 \mathrm{~cm}$ instead of $4.3 \mathrm{~cm}$ ) (Figure 6). Regions 1-7 correspond to the external insulation of the arrester, region 11 to the fiber glass, region 10 to the varistor and regions 8 and 9 to the electrodes. In addition the case that the diameter of the non-linear resistance is higher near the high voltage electrode $(4.8 \mathrm{~cm}$ instead of $4.3 \mathrm{~cm}$ ) (Figure 7) is also examined. Regions 1-7 correspond to the external insulation of the arrester, region 11 to the fiber glass, region 10 to the varistor and regions 8 and 9 to the electrodes.

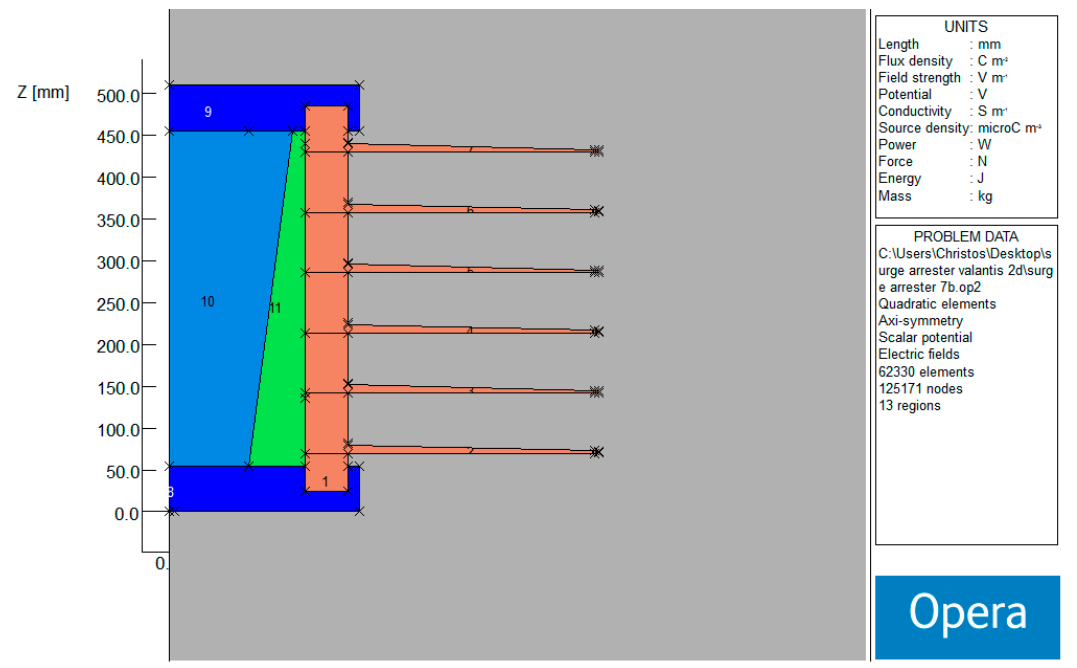

Figure 6. Arrester's model with non uniform increase of the varistor's diameter (case 1).

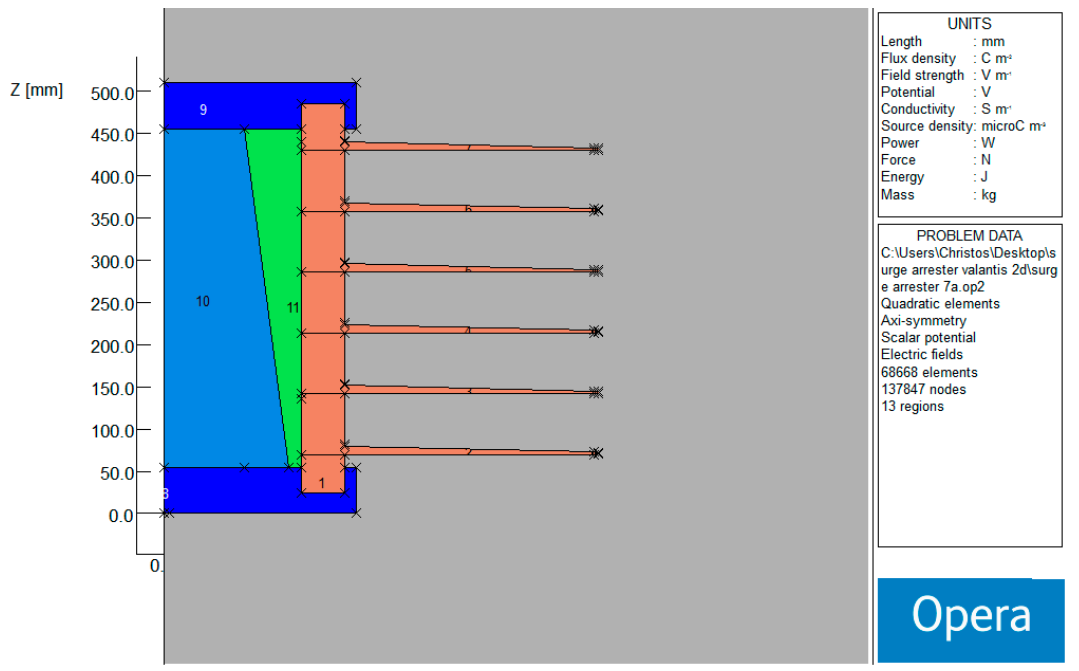

Figure 7. Arrester's model with non uniform increase of the varistor's diameter (case 1).

Figure 8 presents in a common diagram the voltage distribution along the non linear resistance of the arrester for the examined cases. The uniform increase of the diameter of the varistor and case 2 of the non uniform increase of the diameter result in the improvement of the potential distribution, since case 1 of the non uniform increase of the diameter leads to a more intense non uniformity. The simulation and the analysis of the various design proposals, during the initial study of a new arrester's model, is significant and indispensable, in order to obtain a reliable assessment of the electric field around the arrester and the voltage distribution along its non linear resistance. 


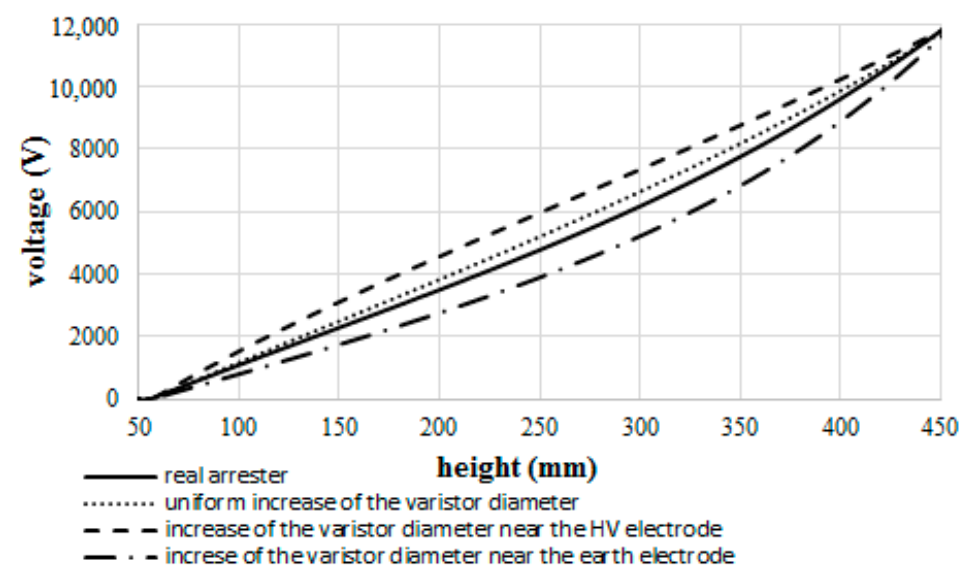

Figure 8. Comparison of the voltage distribution along the non linear resistance for the examined cases.

\section{Special Cases}

\subsection{Pollution on the Surface of the External Insulation of the Arrester}

Surface pollution is one of the main causes of flashover in the external insulations, since it causes the degradation of the insulators, having severe effects in their electric characteristics, and is one of the main reasons of their mis-operation. The pollution, which can be caused by a great variety of sources (sea salt, industries, ash etc.), affects the surface of the insulator and a contaminant layer appears. The wind (mainly), gravity and the electric field are the main bearers of the particles. In addition, by the action of rain, fog, etc. the layer on the surface is dampened and enlarges the conductivity, resulting in an increase of the leakage current, the degradation of their insulating capability, the increase of temperature and the beginning of partial discharges [17]. The above repercussions of the pollution determine the design and the characteristics of the outdoor electrotechnical equipment, exposed to contamination conditions. In order to examine the impact of the pollution on the external surface of the insulator of the arrester under examination, a uniform pollution layer $\left(\varepsilon_{\mathrm{r}}=20, \sigma=10 \mathrm{~S} / \mathrm{m}\right)$ that corresponds to an equivalent salt deposit density (ESDD) C equal to $0.025 \mathrm{mg} / \mathrm{cm}^{2}$, is regarded on the arresters external insulation (Figure 9). Regions 1-7 correspond to the external insulation of the arrester, region 9 to the fiber glass, region 8 to the varistor and regions 10 and 11 to the electrodes.

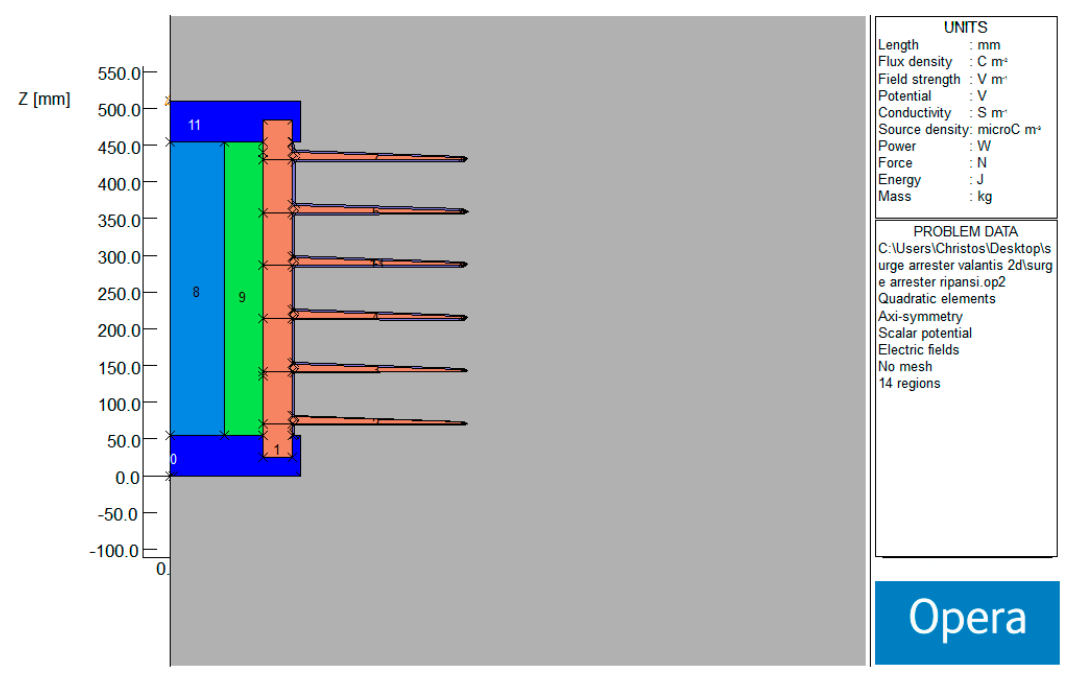

Figure 9. 2D PC Opera model of the arrester under study in the case of pollution on the surface of the external insulation. 
Figures 10 and 11 depict the simulation results for the electric field and the voltage distribution along the varistor considering a $12 \mathrm{kV}$ (phase-to-neutral) applied voltage. The electric field around the arrester is higher compared with the outcomes of a brand new arrester without pollution, since the voltage distribution along the non-linear resistance is more uniform (Figure 18). It is worth mentioning that the electric field is remarkably high near the sheds of the arrester, resulting in increased risk for partial discharges on the external insulation that can lead to deterioration of the insulating material and consequent failure of the arrester.
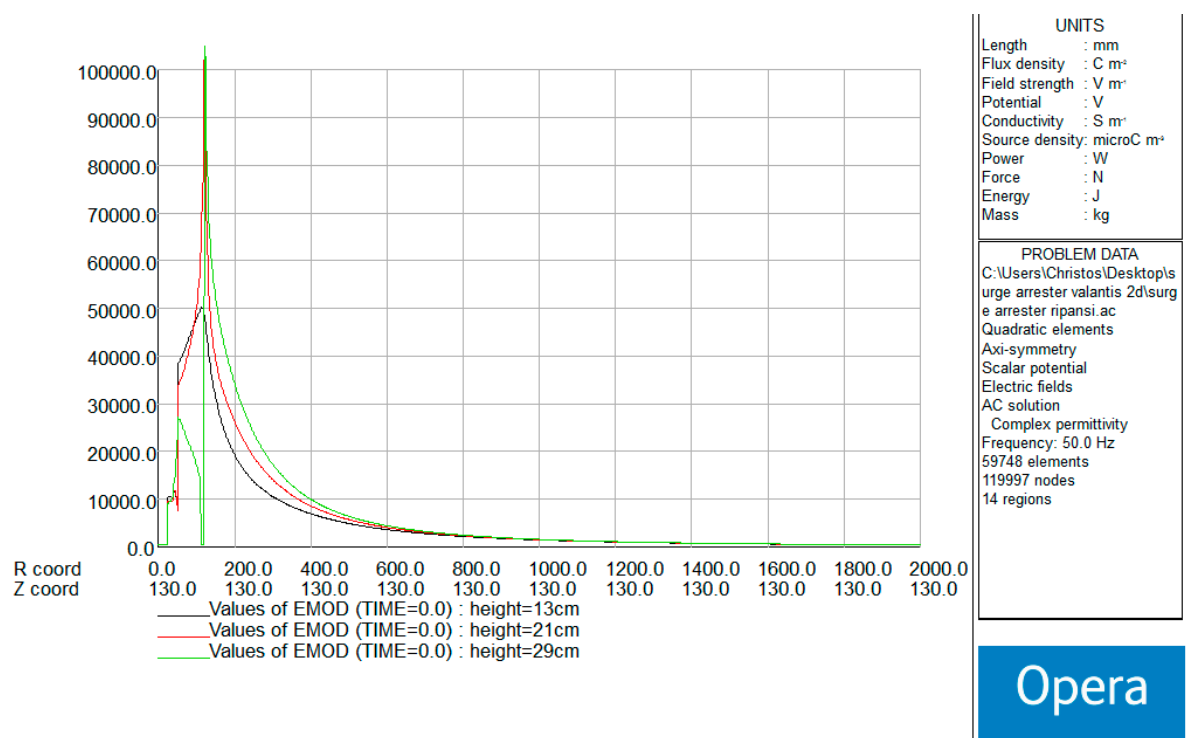

Figure 10. Electric field distribution around the arrester under study in the case of pollution on the surface of the external insulation (2D PC Opera).

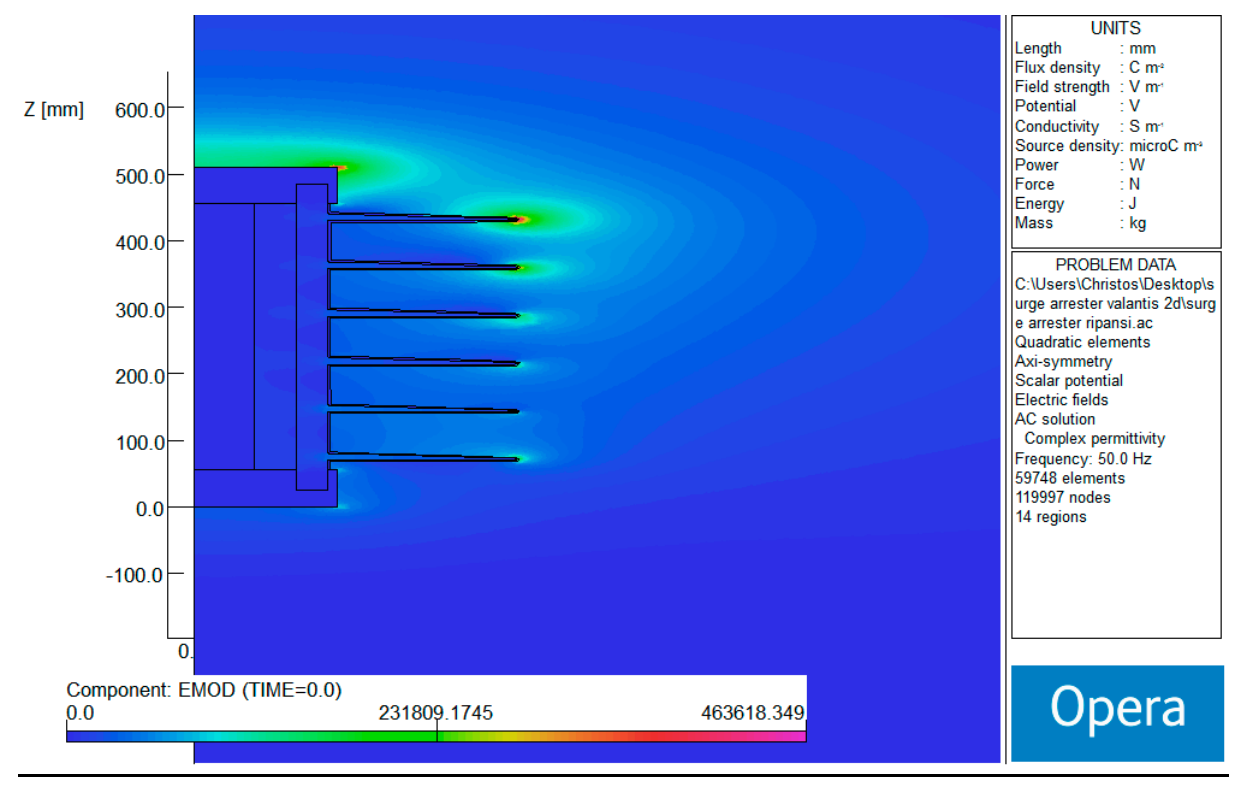

Figure 11. Electric field around the arrester in the case of pollution on the surface of the external insulation (2D PC Opera).

\subsection{Arrester with Broken Shed}

The case that a shed of the arrester is broken is examined. Figure 12 presents the developed 2D PC Opera model, since Figures 13 and 14 depict the extracted outcomes. Note that Regions 1-6 and 11 correspond to the external insulation of the arrester, region 8 to the fiber glass, region 7 to the varistor 
and regions 9 and 10 to the electrodes. The broken shed does not affect the voltage distribution along the varistor (Figure 18), but results in stress of the device due to the increased electric field.

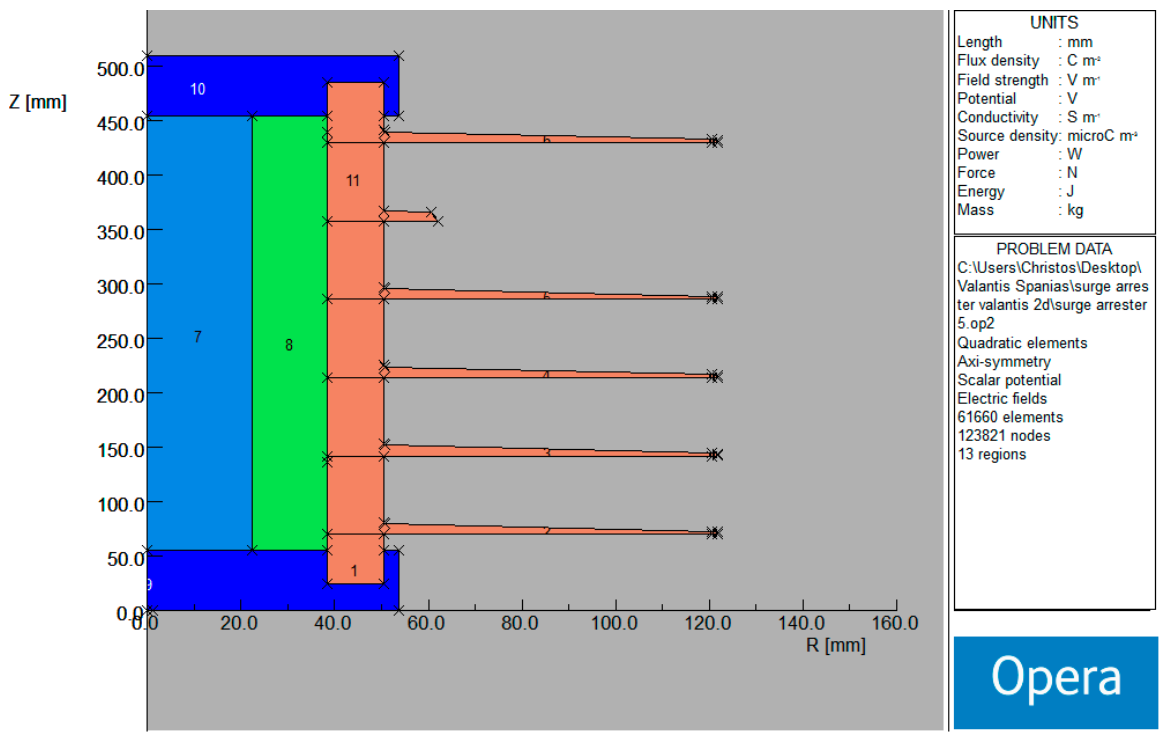

Figure 12. 2D PC Opera model of the arrester under study in the case of a broken shed.

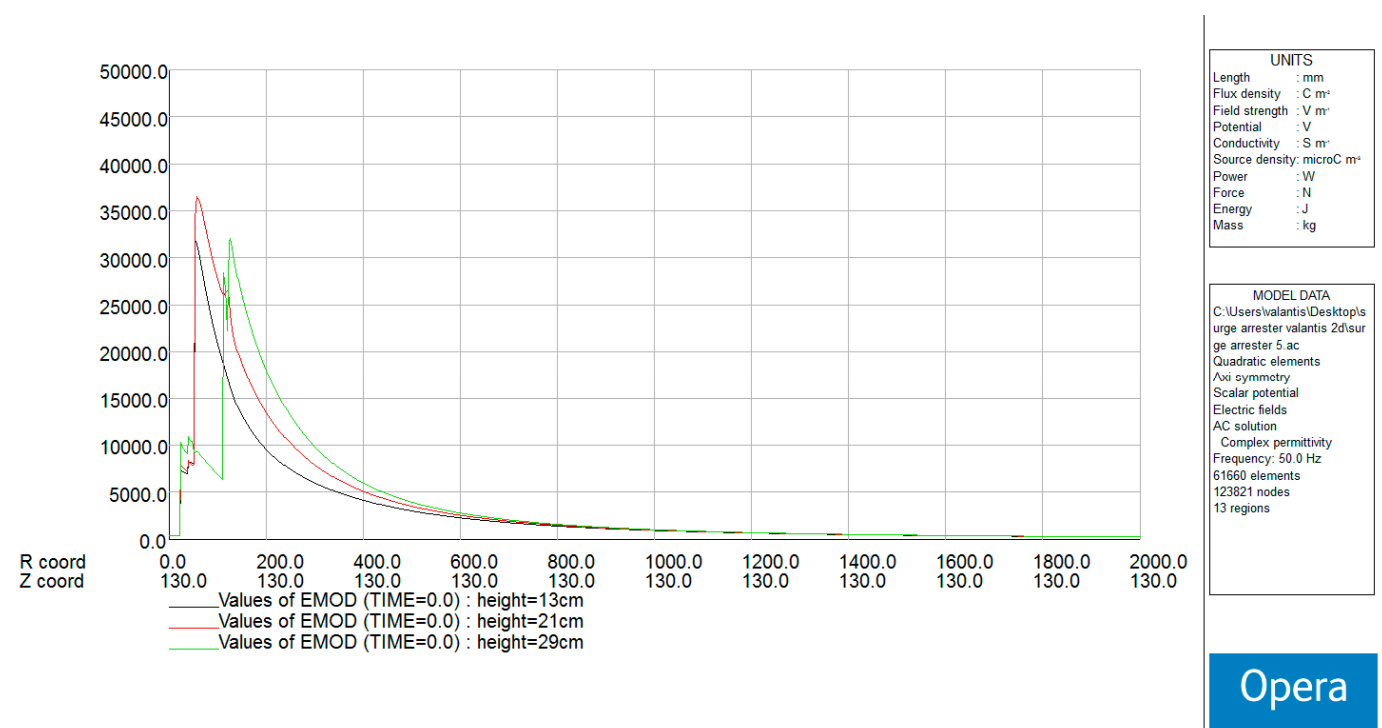

Figure 13. Electric field distribution around the arrester under study in the case of a broken shed (2D PC Opera). 


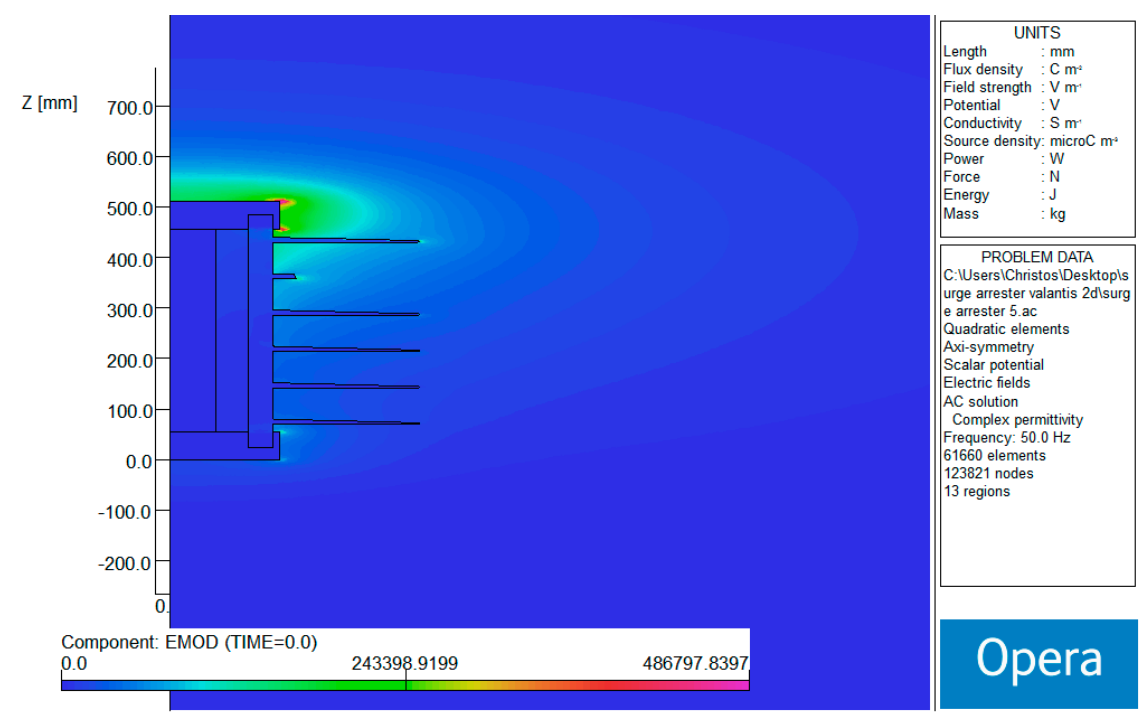

Figure 14. Electric field around the arrester in the case of a broken shed (2D PC Opera).

\section{Discussion}

Figures 15-17 present in a common diagram for each height (from the earth electrode) the estimated results of the electric filed in function with the distance from the arrester under study. The electric field is affected by the geometry of the equipment and the electrical characteristics of the materials. Pollution affects the electrical characteristics of the external surface of the arrester, since broken sheds alter the geometry of the arrester under study. The above factors influence the developed electric field around the arrester. Consequently, the presence of pollution on the surface of the external insulation of the arrester results in a higher value of the electric field. Moreover, the computed electric field is more intense near the high voltage electrode and is reduced as the distance from the arrester is increased.

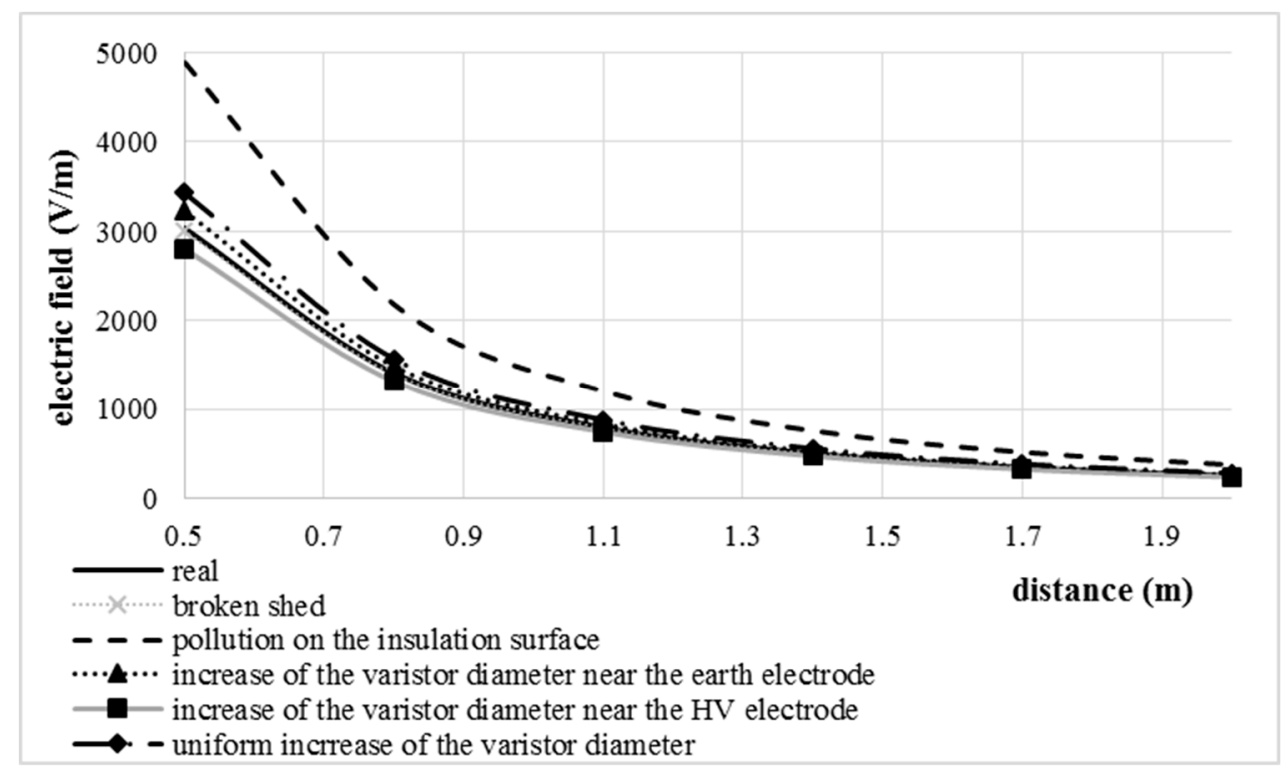

Figure 15. Electric field $(\mathrm{V} / \mathrm{m})$ in function with the distance from the arrester (height from the earth electrode equal to $13 \mathrm{~cm}$ ). 


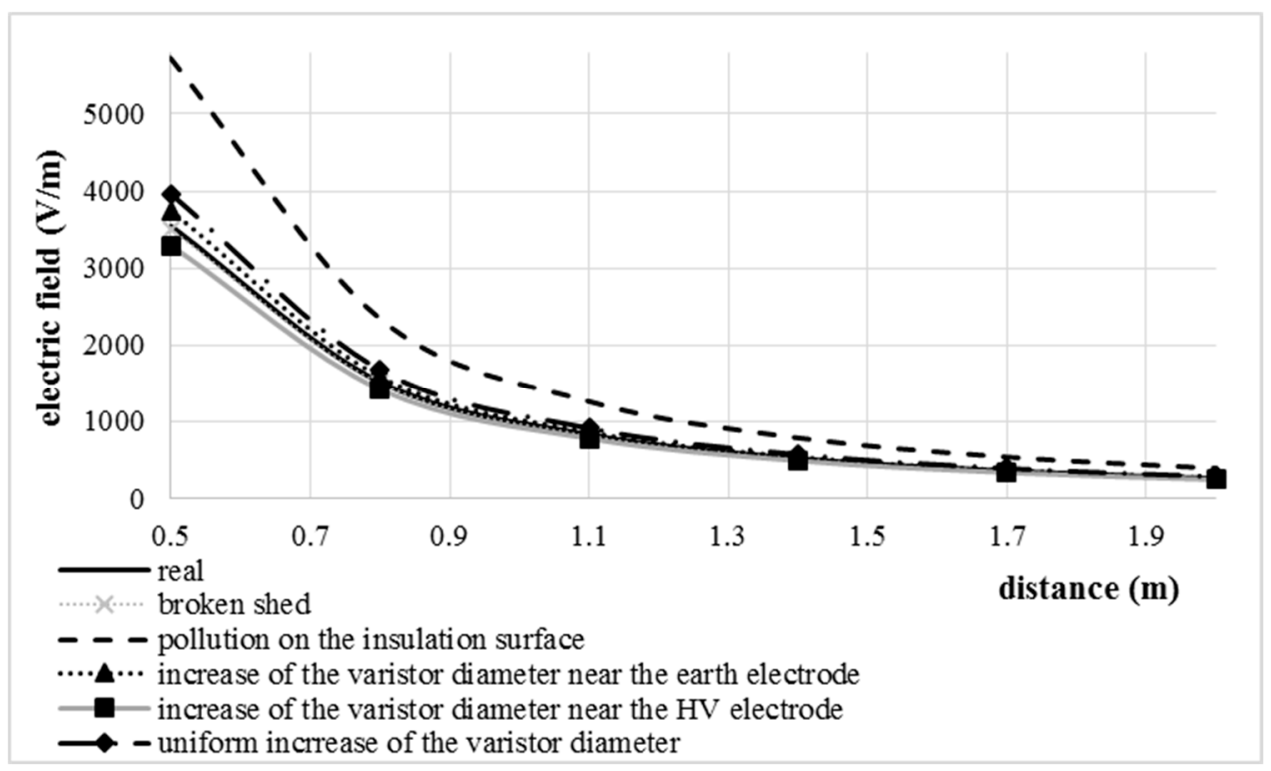

Figure 16. Electric field $(\mathrm{V} / \mathrm{m})$ in function with the distance from the arrester (height from the earth electrode equal to $21 \mathrm{~cm})$.

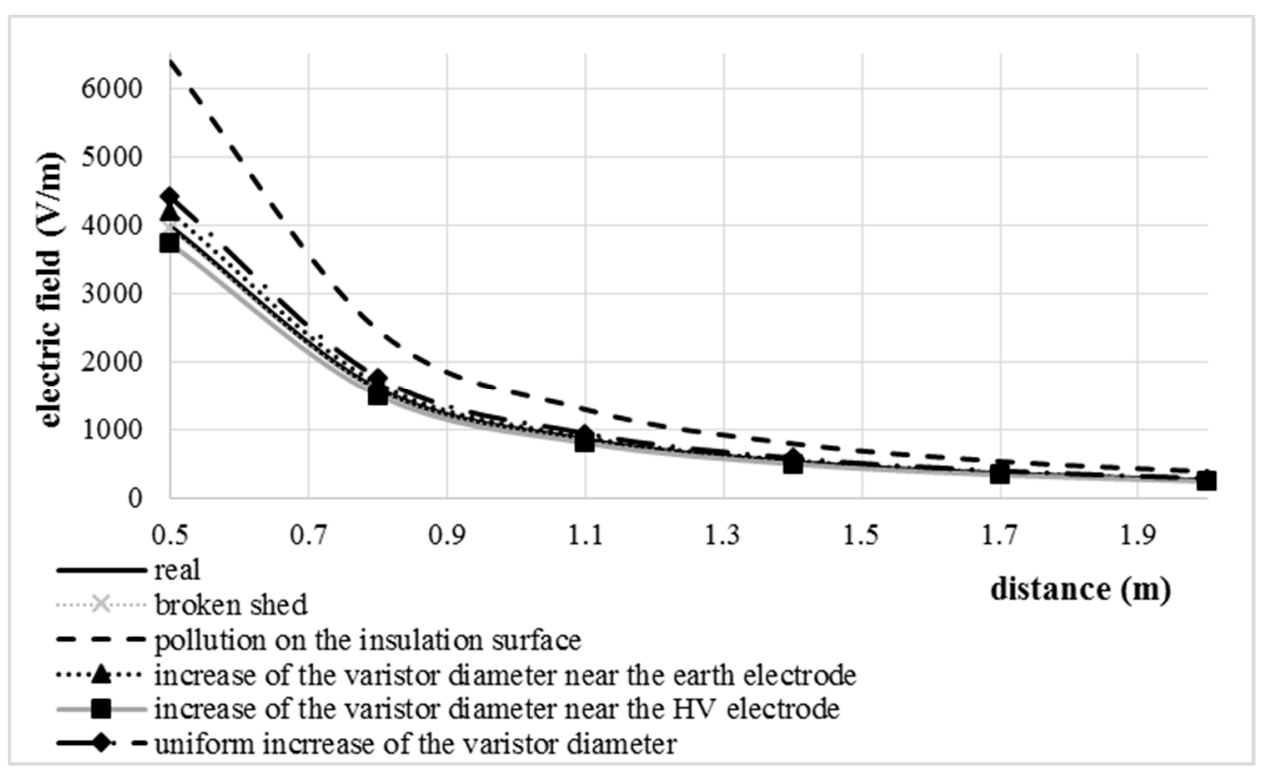

Figure 17. Electric field $(\mathrm{V} / \mathrm{m})$ in function with the distance from the arrester (height from the earth electrode equal to $29 \mathrm{~cm}$ ).

Figure 18 depicts the voltage distribution along the non-linear resistance of the arrester for all the examined cases. The increase of the uniform increase of the varistor diameter or the increase near the high voltage electrode result in improvement of the voltage distribution, mitigating the repercussions of the constant strain of the nonlinear resistance that could downgrade the V-I characteristic of the varistor. In the case of pollution on the surface of the external insulation the voltage distribution is also becomes uniform (this case cannot be deployed due to the downgrade of the insulating characteristics of the device). 


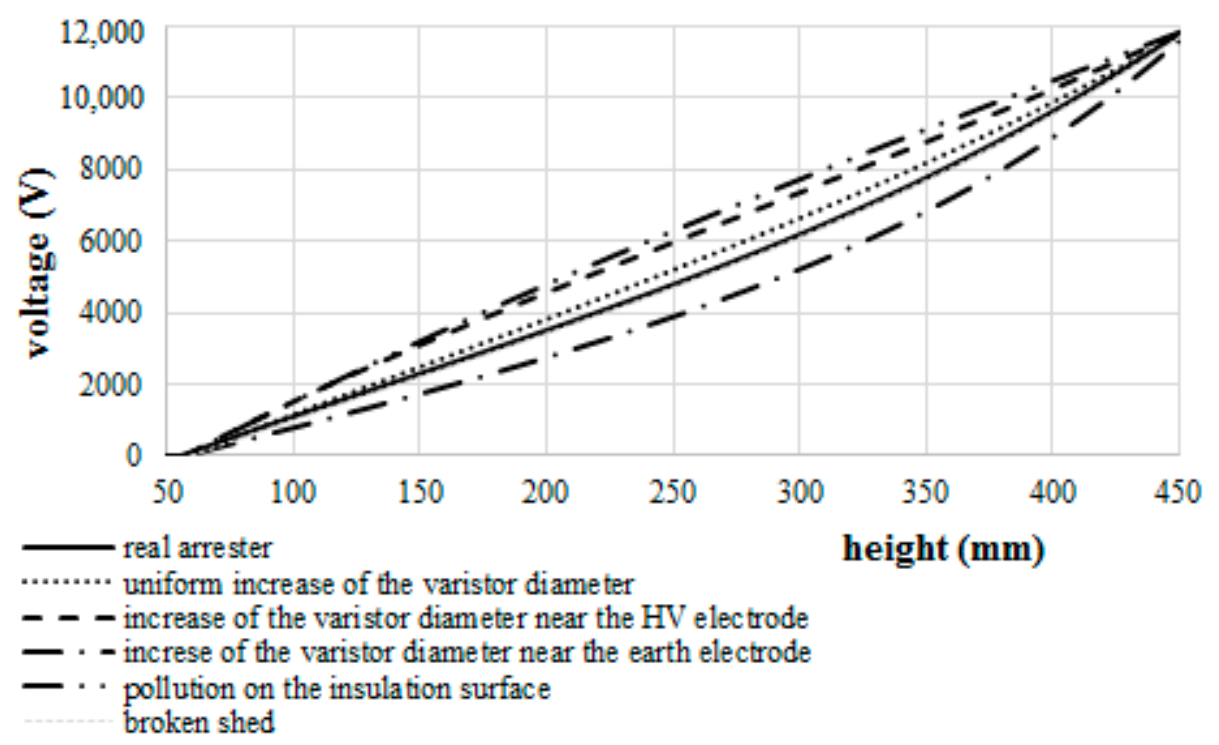

Figure 18. Comparison of the voltage distribution along the non linear resistance for the examined cases of sections 4 and 5 .

\section{Conclusions}

Surge arresters, offering protection to the electrical equipment against lightning overvoltages, constitute one of the most important parts of the distribution network as direct or indirect surges can create serious problems and malfunctions to the electrical system. The voltage distribution along the non linear resistance of medium voltage gapless surge arresters is an issue of great importance for their efficient operation and, consequently, the protection of the electric network. The improvement of the voltage distribution along the varistor is required, in an effort to achieve the optimum utilization of the materials and to mitigate the arising damages due to the excessive stresses.

In previous works, 2D and 3D simulation models were developed to estimate the electric field around a medium voltage arrester. The theoretical results were confirmed by comparison with laboratory measurements, presenting sufficient agreement; the small difference between the PC Opera and the laboratory outcomes prove that the proposed simulation methodology is a useful and reliable tool for the estimation of the voltage along the varistor.

Considering that the equipment or the place for experiments are usually not available, the current work proposes the use of a simulation tool that extracts precise results in a fast and economic way. In details, the two designed, in PC Opera, models can be applied for the computation of the voltage distribution along the varistor, since there is no practical method to measure it. The extracted results show that the voltage distribution can be easily and reliably estimated, if the desirable geometry is designed with precision, by using appropriate computer tool.

According to the above, this paper examines new configurations for the improvement of the voltage distribution and the impact of various factors (pollution on the surface of the external insulation, broken sheds) on the voltage distribution. The extracted results can contribute to the more efficient design of modern metal oxide gapless surge arresters, in an effort to ensure their reliable operation to protect the electrical equipment against lightning surges.

Author Contributions: All authors have equally contributed in this work.

Funding: This research received no external funding.

Conflicts of Interest: The authors declare no conflict of interest. 


\section{References}

1. Latiff, N.A.A.; Illias, H.; Bakar, A.H.A.; Dabbak, S.Z.A. Measurement and modelling of leakage current behaviour in $\mathrm{ZnO}$ surge arresters under various applied voltage amplitudes and pollution conditions. Energies 2018, 11, 875. [CrossRef]

2. Vahidi, B.; Nasab, R.S.; Moghani, J.Sh.; Kashi, S.A.; Hosseinian, S.H. Three dimensional analyses of electric field and voltage distribution on ZnO surge arrester with broken sheds. In Proceedings of the 2005 IEEE/PES Transmission \& Distribution Conference \& Exposition: Asia and Pacific, Dalian, China, 18 August 2005.

3. Christodoulou, C.A.; Spanias, C.A.; Kontargyri, V.T.; Gonos, I.F.; Stathopulos, I.A. Study of the electric field around a metal oxide surge arrester: Measurement and simulation. High Voltage Eng. 2013, 39, $21460-21467$.

4. Chrzan, K.L.; Gielniak, J. Voltage distribution along metal oxide surge arresters. In Proceedings of the XIII International Symposium on High Voltage Engineering, Delft, The Netherlands, 25-29 August 2003.

5. Hinrichsen, H. Diagnostics and monitoring of metal-oxide surge arresters in high-voltage networks-comparison of existing and newly developed procedures. IEEE Trans. Power Deliv. 2001, $16,138-143$.

6. $\quad$ Surge Arresters_Part 4: Metal-Oxide Surge Arresters without Gaps for A. C. Systems, 2nd ed.; National Standards Authority of Ireland (NSAI): Dublin, Ireland, 2004.

7. Karthik, R. A novel analysis of voltage distribution in Zinc Oxide arrester using finite element method. Int. J. Recent Trends Eng. 2009, 1, 1-3.

8. Tighilt, F.; Bayadi, A. Computed voltage distribution in $\mathrm{ZnO}$ arrester under pollution by finite element method. In Proceedings of the 4th International Conference on Computer Integrated Manufacturing CIP'2007, Setif, Algeria, 3-4 November 2007; p. E24.

9. Vahidi, B.; Shariati Nasab, R.; Moghani, J.S. Analysis of electric field and voltage distribution Zno surge arrester for polluted condition. In Proceedings of the XIV International Symposium on High Voltage Engineering, Tsinghua University, Beijing, China, 25-29 August 2005; p. A-51.

10. Meshkatoddini, M. Study of the electric field intensity in bushing integrated ZnO surge arresters by means of finite element analysis. In Proceedings of the COMSOL Users Conference, Boston, MA, USA, 7 November 2006.

11. Kumar, U.; Mogaveera, V. Voltage distribution studies on ZnO arresters. IEE Gener. Transm. Distrib. 2002, 149, 457-462. [CrossRef]

12. Han, S.J.; Zou, J.; Gu, S.Q.; He, J.L.; Yuan, J.S. Calculation of the potential distribution of high voltage metal oxide arrester by using an improved semi-analytic Finite Element Method. IEEE Trans. Magn. 2005, 41, 1392-1395. [CrossRef]

13. Spanias, C.A.; Christodoulou, C.A.; Gonos, I.F.; Stathopulos, I.A. Electric field measurements around a metal oxide surge arrester. In Proceedings of the International Conference on High Voltage Engineering and Application (ICHVE 2010), New Orlean, LA, USA, 11-14 October 2010.

14. Vita, V.; Christodoulou, C.A. Comparison of ANN and finite element analysis simulation software for the calculation of the electric field around metal oxide surge arresters. Elec. Power Syst. Res. 2016, 133, 87-92. [CrossRef]

15. OPERA-2d Reference Manual[R]; Vector Fields Limited: Kidlington, UK, 1999.

16. OPERA-3d Reference Manual[R]; Vector Fields Limited: Kidlington, UK, 2004.

17. Hernanz, J.R.; Martin, J.C. Insulator pollution in transmission lines. Renew. Energy Power Qual. J. 2006, 1, 124-130. [CrossRef]

(C) 2018 by the authors. Licensee MDPI, Basel, Switzerland. This article is an open access article distributed under the terms and conditions of the Creative Commons Attribution (CC BY) license (http:/ / creativecommons.org/licenses/by/4.0/). 